\title{
Pretreatment resistance mutations and treatment outcomes in adults living with HIV-1: a cohort study in urban Malawi
}

\author{
F. Neuhann ${ }^{1^{*}+}$ D, A. de Forest ${ }^{1 \dagger}$, E. Heger ${ }^{2}$, A. Nhlema ${ }^{3}$, C. Scheller ${ }^{4}$, R. Kaiser ${ }^{2}$, H. M. Steffen ${ }^{5}$, H. Tweya ${ }^{3}$, \\ G. Fätkenheuer ${ }^{6}$ and S. Phiri $3,7,8,9$
}

\begin{abstract}
Background: Pre-treatment drug resistance (PDR) among antiretroviral drug-naïve people living with HIV (PLHIV) represents an important indicator for the risk of treatment failure and the spread of drug resistant HIV variants. We assessed the prevalence of PDR and treatment outcomes among adults living with HIV-1 in Lilongwe, Malawi.

Methods: We selected 200 participants at random from the Lighthouse Tenofovir Cohort Study (LighTen). Serum samples were drawn prior to treatment initiation in 2014 and 2015, frozen, and later analyzed for the presence of HIV-1 drug resistance mutations. Amplicons were sequenced and interpreted by Stanford HIVdb interpretation algorithm 8.4. We assessed treatment outcomes by evaluating clinical outcome and viral suppression at the end of the follow-up period in October 2019.

Results: PDR testing was successful in 197 of 200 samples. The overall NNRTI- PDR prevalence was 13.7\% (27/197). The prevalence of intermediate or high level NNRTI- PDR was 11.2\% (22/197). The most common mutation was K103N (5.6\%, 11/197), followed by Y181C (3.6\%, 7/197). In one case, we detected an NRTI resistance mutation (M184V), in combination with multiple NNRTI resistance mutations. All HIV-1 isolates analyzed were of subtype C. Of the 27 patients with NNRTI- PDR, 9 were still alive, on ART, and virally suppressed at the end of follow-up.

Conclusion: The prevalence of NNRTI- PDR was above the critical level of $10 \%$ suggested by the Global Action Plan on HIV Drug Resistance. The distribution of drug resistance mutations was similar to that seen in previous studies from the region, and further supports the introduction of integrase inhibitors in first-line treatment in Malawi. Furthermore, our findings underline the need for continued PDR surveillance and pharmacovigilance in Sub-Saharan Africa.
\end{abstract}

Keywords: HIV, Drug resistance mutations, Pretreatment resistance, Non-nucleoside reverse transcriptase, Malawi

\section{Introduction}

The global availability of antiretroviral therapy (ART) has resulted in a great reduction of new HIV infections, HIV related morbidity, and mortality $[1,2]$. However, settings with the highest prevalence of HIV often lack critical

\footnotetext{
*Correspondence: florian.neuhann@uni-heidelberg.de

${ }^{\dagger} \mathrm{F}$ Neuhann, A de Forest contributed equally to this work

${ }^{1}$ Heidelberg Institute for Global Health, University Hospital of Heidelberg, Im Neuenheimer Feld 130.3, 69120 Heidelberg, Germany

Full list of author information is available at the end of the article
}

resources, including infrastructure for monitoring the development of HIV drug resistance mutations. In lowincome countries, drug resistance surveillance is only performed periodically at specific sites or in populations under treatment, and rarely prior to treatment initiation. The development and spread of HIV drug resistance (HIVDR) could endanger treatment success, and ultimately threaten the control of the epidemic [3].

Pretreatment drug resistance (PDR) is defined by the WHO as resistance that is detected among people either newly initiating or reinitiating first-line ART [3]. In

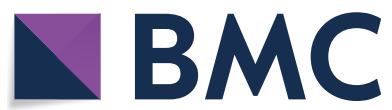

(c) The Author(s) 2020. This article is licensed under a Creative Commons Attribution 4.0 International License, which permits use, sharing, adaptation, distribution and reproduction in any medium or format, as long as you give appropriate credit to the original author(s) and the source, provide a link to the Creative Commons licence, and indicate if changes were made. The images or other third party material in this article are included in the article's Creative Commons licence, unless indicated otherwise in a credit line to the material. If material is not included in the article's Creative Commons licence and your intended use is not permitted by statutory regulation or exceeds the permitted use, you will need to obtain permission directly from the copyright holder. To view a copy of this licence, visit http://creativeco mmons.org/licenses/by/4.0/. The Creative Commons Public Domain Dedication waiver (http://creativecommons.org/publicdomain/ zero/1.0/) applies to the data made available in this article, unless otherwise stated in a credit line to the data. 
previous studies, non-nucleoside reverse transcriptase inhibitor (NNRTI) related PDR exceeded $10 \%$ in many African settings, supporting the move to include the integrase inhibitor dolutegravir (DTG) in first-line regimens $[4,5]$.

Malawi has set up an effective HIV treatment program. According to the Malawian Ministry of Health quarterly reports, as of September 2018, out of the total one million PLHIV in Malawi, around 94\% knew their status, $80 \%$ were on ART, and $89 \%$ of those on ART were virally suppressed [6].

The Lighthouse Clinic is the largest provider of HIV counselling, treatment and care in Lilongwe, Malawi [7]. We commenced the Lighthouse Tenofovir Cohort Study (LighTen; ClinicalTrials.gov NCT02381275) in August 2014. We enrolled 1432 ART-naïve adults living with HIV and followed them from initiation with tenofovirbased ART for a period of 36 months, with the primary objective of analyzing changes in kidney function. At the onset of the study, the Lighthouse clinic was providing comprehensive HIV services to over 24000 PLHIV in greater Lilongwe (own data). We report the frequency and pattern of pretreatment HIV-1 drug resistance (PDR) and the treatment outcomes among a subgroup of this cohort.

\section{Methods}

\section{Setting}

The study was conducted at one of the Lighthouse clinics on the campus of the Kamuzu Central Hospital in Lilongwe, Malawi. The Lighthouse is a large, specialized center for HIV treatment and care in the central region of Malawi, and mainly serves an urban population of lower socio-economic status. Access to testing and counselling as well as treatment and care is free of charge at the point of delivery [7].

\section{Participants}

The present study is a subgroup analysis of participants in the LighTen study, which enrolled 1432 ART-naïve adults aged 18 years or older. 200 consecutively enrolled patients were selected at random for PDR testing during the recruitment process in 2014 and 2015. We drew baseline serum samples prior to treatment initiation, stored them at minus $80^{\circ} \mathrm{C}$, and later analyzed them for the presence of HIV-1 drug resistance mutations.

All LighTen enrollees received first-line ART according to Malawian treatment guidelines, which at the time of enrolment consisted of lamivudine (3TC), tenofovir disoproxil fumarate (TDF) and efavirenz (EFV). Decisions on treatment regimen and potential switches were not informed by the results of drug resistance testing. Participants were seen regularly during routine follow-up visits as scheduled in the Malawian national treatment guidelines and according to clinical needs. Treatment outcomes were analyzed at the end of 2019.

\section{Laboratory processes}

All participants had baseline evaluation beyond the standards of the Malawian HIV treatment program, including full blood count, liver and renal function tests, CD4 cell count, and HIV viral load testing (see LighTen protocol; ClinicalTrials.gov NCT02381275).

Nucleic acid extraction from $500 \mu \mathrm{L}$ of serum was performed using the DNA and viral NA large volume kit (Roche Diagnostics, Mannheim, Germany) for the automated MagNA Pure 96 system (Roche Diagnostics, Rotkreuz, Switzerland). The protease and reverse transcriptase regions were amplified for resistance analysis as described by Lübke et al. [8], and the envelope region for tropism determination as described by Sierra et al. [9]. For HIV subtyping, the COMET tool version 2.2 [10] was used. Amplicons were sequenced by Next Generation Sequencing using Illumina Sequencing Technology (Illumina Inc., San Diego, USA) and interpreted by Stanford HIV database interpretation algorithm 8.4 (HIVdb) [11].

\section{Outcomes}

Outcome variables were: viral load 6 months after treatment initiation, last viral load, and treatment outcome at the time of assessment. Treatment outcomes were categorized as either: alive and on treatment at the Lighthouse clinic, transferred out to another treatment facility, stopped ART, died, changed ART regimen, withdrawn from the study, or defaulted. The status "defaulted" was assigned to patients who had not been in contact with the clinic 60 days after a missed follow-up appointment. Drug resistance mutations conferring at least low-level resistance according to the Stanford HIVdb [9] were counted, and the treatment outcomes of affected patients were analyzed.

\section{Statistical methods}

We compared the group included in drug resistance testing to the cohort not included in resistance testing at baseline using Chi square, Student's Test and KruskallWallis test as appropriate for the type of variable and the respective distribution. The significance level was set at 0.05 .

\section{Ethics}

The LighTen study protocol was approved by the Ethics Committee of the National Health Research Committee of the Ministry of Health, Malawi and the ethics committees of the Universities of Heidelberg and Cologne, Germany. 


\section{Results}

Overall, LighTen enrolled 1432 participants, of whom 200 were included in the HIV-1 resistance testing group. HIVDR testing was successful in 197 of 200 samples. All analyzed HIV-1 isolates were of subtype C.

Baseline characteristics of the HIVDR testing group differed from the total LighTen cohort, with a higher proportion of WHO stage 1 (65\% vs 43\%) and a lower proportion of WHO stage $3(9.6 \%$ vs $34.7 \%)$ in the HIVDR testing group. The HIVDR testing group had a higher median viral load. The groups also differed significantly regarding the treatment outcome "Alive and on ART" (Table 1).

The overall NNRTI-PDR prevalence was $13.7 \%$ (27/197). The prevalence of mutations conferring intermediate or high level resistance to first-line ART was $11.2 \%(22 / 197)$. The most common PDR was K103N $(5.6 \%, 11 / 197)$, followed by Y181C (3.6\%, 7/197). In one case, we detected an additional NRTI drug resistance mutation (M184V) (Table 2). We identified the accessory mutation E138A in eight samples.
Of the 27 individuals with NNRTI- PDR mutations, 11 were still alive and on treatment at Lighthouse at the end of follow-up, 9 of whom were virally suppressed. Of the 16 patients not alive and on treatment at the end of follow-up, 12 had defaulted, two had transferred to another clinic and two had died (see Fig. 1). A synopsis of the treatment outcomes is provided in Table 3.

\section{Discussion}

We analyzed baseline samples from 197 participants consecutively enrolled in the LighTen cohort study. The prevalence of treatment relevant PDR in our sample reached $11.2 \%$, almost exclusively affecting the NNRTI class. Since the Malawian HIV treatment guideline currently does not include resistance testing for ART-naïve patients prior to ART initiation, all patients initially received the standard first-line treatment of 3TC/TDF/ EFV.

Although the HIVDR testing group differed significantly from the overall LighTen cohort in some baseline variables and treatment outcomes, these differences do not suggest a significant bias that would affect the level

Table 1 Comparison of participants with and without HIVDR testing

\begin{tabular}{|c|c|c|c|}
\hline & \multicolumn{2}{|l|}{ Participants } & \multirow[t]{2}{*}{$\mathbf{P}$} \\
\hline & No HIVDR testing & HIVDR testing & \\
\hline$n$ & 1235 & 197 & \\
\hline \multicolumn{4}{|l|}{$\operatorname{Sex}(\%)$} \\
\hline Female & $692(56.0)$ & $122(61.9)$ & $0.140^{\mathrm{a}}$ \\
\hline Male & $543(44.0)$ & $75(38.1)$ & \\
\hline Age [mean (SD)] & $36.20(9.31)$ & $35.09(9.11)$ & $0.118^{b}$ \\
\hline BMI [mean (SD)] & $24.28(4.91)$ & $23.93(4.63)$ & $0.348^{b}$ \\
\hline \multicolumn{4}{|l|}{ WHO stage (\%) } \\
\hline 1 & $531(43.0)$ & $128(65.0)$ & $<0.001^{\mathrm{a}}$ \\
\hline 2 & $183(14.8)$ & $38(19.3)$ & \\
\hline 3 & $429(34.7)$ & $19(9.6)$ & \\
\hline 4 & $92(7.4)$ & $12(6.1)$ & \\
\hline CD4 count (median [IQR]) & $269.5[125 ; 420]$ & $247.5[89 ; 420]$ & $0.230^{c}$ \\
\hline Viral load (median [IQR]) & 33000 [6 696; 140 844] & 112599 [21 318; 454638$]$ & $<0.001^{c}$ \\
\hline \multicolumn{4}{|l|}{ Outcome (\%) } \\
\hline Alive on ART & $715(57.9)$ & $119(60.4)$ & $0.020^{\mathrm{a}}$ \\
\hline Defaulted & $267(21.6)$ & $32(16.2)$ & \\
\hline Transferred out & $118(9.6)$ & $21(10.7)$ & \\
\hline Changed ART regimen & $62(5.0)$ & $3(1.5)$ & \\
\hline Died & $41(3.3)$ & $14(7.1)$ & \\
\hline Withdrawn & $27(2.2)$ & $7(3.6)$ & \\
\hline Stopped ART & $5(0.4)$ & $1(0.5)$ & \\
\hline
\end{tabular}

CD4 count: number of CD-4 positive T-cells per $\mu$ l; viral load: number of copies of HIV-1 RNA per ml of Serum

$n$ number of participants, $S D$ standard deviation, $I Q R$ interquartile range, HIVDR HIV-1 drug resistance mutation, $B M I$ body mass index, $\mathrm{kg} / \mathrm{m}^{2}$, WHO stage World Health Organization stage of clinical HIV illness

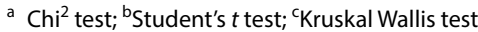


Table 2 Overview and frequency of identified mutations in 27 patients (potentially treatment relevant mutations in italic)

\begin{tabular}{llll}
\hline Identified & mutations $(\mathbf{n})$ & & \\
\hline NRTI & Accessory PI & NNRTI & Resistance level to EFV \\
\hline M184 (1) & T47S (7) & K103N (11) & High \\
& Q58E (4) & A98G (3) & $\begin{array}{l}\text { High level resistance 3TC, } \\
\text { Low level resistance EFV }\end{array}$ \\
& K20T (1) & V106M (2) & High \\
M46L (1) & Y181C (7) & Intermediate \\
M46V (1) & G190A (2) & Intermediate \\
N88D (1) & K103S (1) & Intermediate \\
& K238T (1) & Intermediate \\
& H221Y (4) & Low \\
& & V108I (4) & Low \\
& E138G (2) & Low \\
& V179D (2) & Low \\
& E138K (1) & Low \\
& K101E (1) & Low
\end{tabular}

The total number of mutations reported is higher than the number of individual samples with NNRTI-DRMs, as many samples showed multiple mutations

NRTI nucleoside/nucleotide reverse transcriptase inhibitors, PI protease inhibitors, NNRTI non-nucleoside reverse-transcriptase inhibitors, EFV efavirenz, 3TC lamivudine

a According to the Stanford Drug Resistance Database and pattern of PDR. There was a higher proportion of clients in earlier stages of HIV disease (lower WHO stage, higher viral load) in the HIVDR testing group.

The results of HIVDR testing could not influence the choice of treatment, as testing was performed retrospectively from stored samples. Among the 12 patients with $\mathrm{K} 103 \mathrm{~N}$ and/or V106M mutations (leading to a functional dual NRTI-therapy), only four were alive and on ART at the end of follow-up. Remarkably, two of these four patients were still on first-line treatment and virally suppressed. Our findings are in line with the multi-centre cohort study by Hamers et al., which found an odds ratio of 2.13 for virological failure in patients with PDR to at least one prescribed drug [12].

Our results echo other data from the region. In a cohort of Malawians living with HIV, Rutstein et al. reported the same proportion of 11\% NNRTI-PDR among 46 acutely infected persons in Malawi, with a similar distribution of different sub-types of mutations [13].

According to recent data from the Malawian population-based HIV impact assessment consortium, the overall level of viral suppression in Malawi is $89 \%$, with considerable variation between different regions in Malawi [14] For the central region, where this study

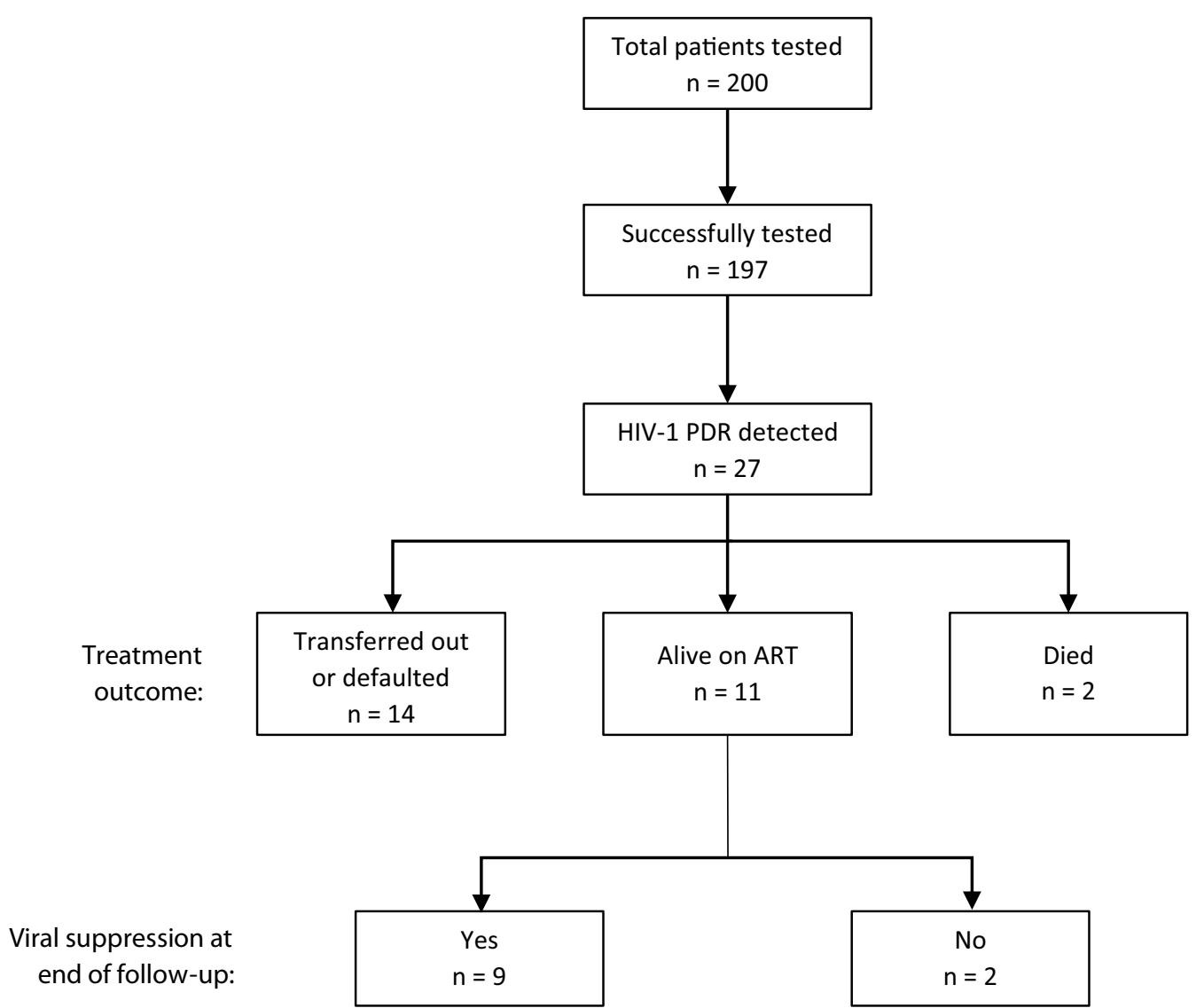

Fig. 1 Flowchart of HIV-1 pretreatment drug resistance testing and treatment outcomes. PDR HIV-1 pretreatment drug resistance, ART antiretroviral therapy. For details on treatment outcome categories, see text 


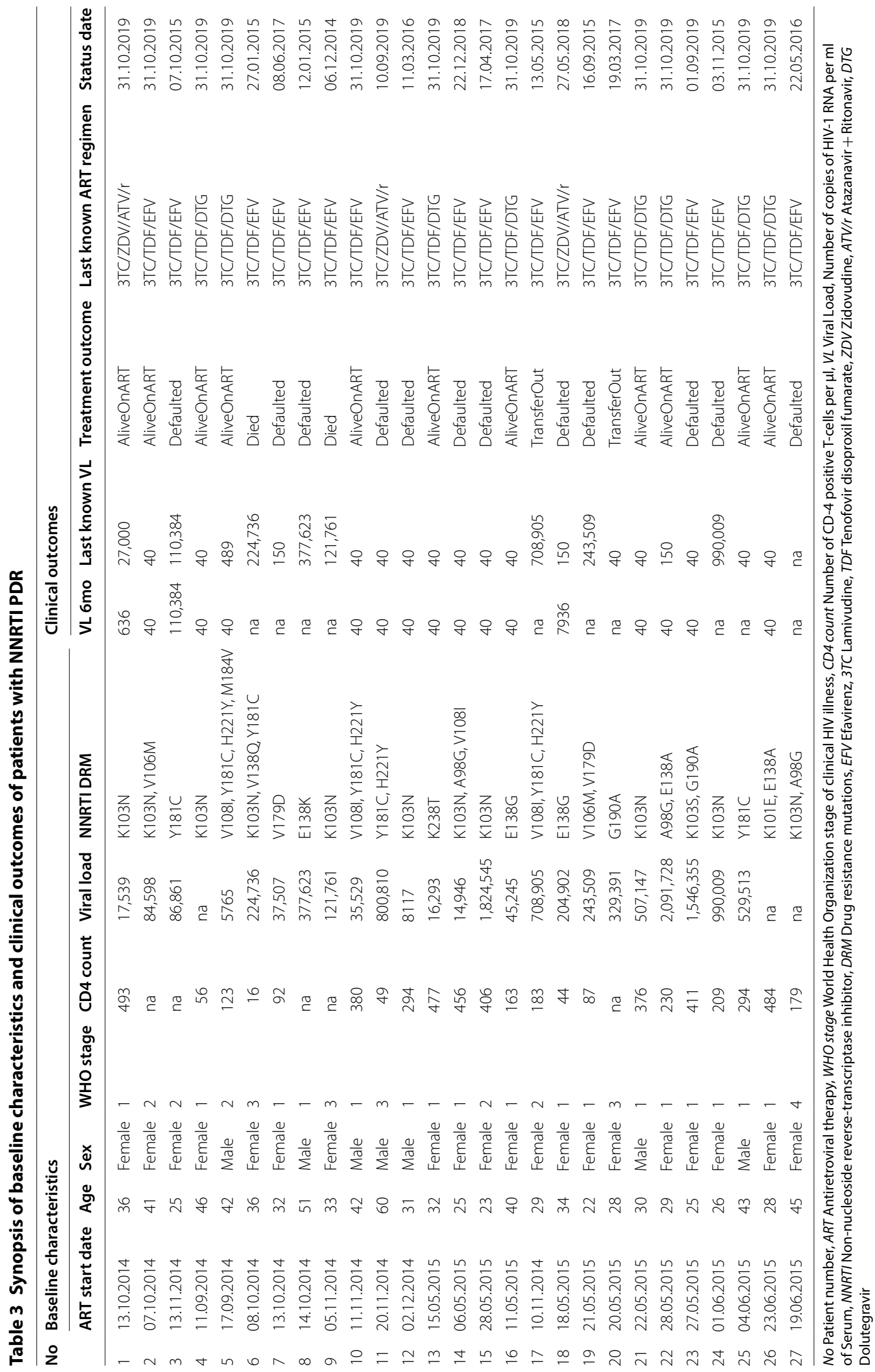


was conducted, the data report proportions of treated patients with suppressed viral load between 64.9\% (Lilongwe City) and 70.6\% (Central West Region). [14] Our data raise the question whether differences in virological outcomes may be associated with different regional levels of PDR. Fortunately, all HIV-1 isolates analyzed here expressed phenotypes predicted to be sensitive to Malawi's second-line treatment options.

Our observations add to the body of HIV-1 drug resistance data from Southern Africa and are in line with other reports from the region $[15,16]$. The number of observations is higher than previous results from Sub-Saharan Africa, and our results include treatment outcomes of the patients.

\section{Conclusion}

The prevalence of NNRTI-PDR was above the critical level of $10 \%$ suggested by the Global Action Plan on HIV Drug Resistance [16]. The study findings support the introduction of integrase inhibitors (i.e. dolutegravir) [17] in first-line treatment in Malawi. Furthermore, they underline the need for continued resistance surveillance and pharmacovigilance in Sub-Saharan Africa.

\begin{abstract}
Abbreviations
3TC: Lamivudine; ART: Antiretroviral therapy; DTG: Dolutegravir; DRM: Drug resistance mutation; EFV: Efavirenz; HIVDR: HIV drug resistance; LighTen: Lighthouse Tenofovir Cohort Study; NNRTI: Non-nucleoside reverse transcriptase inhibitor; NRTI: Nucleoside/nucleotide reverse transcriptase inhibitors; PDR: Pretreatment drug resistance; TDF: Tenofovir disoproxil fumarate; WHO: World Health Organization.
\end{abstract}

\section{Acknowledgements \\ The authors would like to acknowledge the support by Lighthouse study nurses and clinicians, in particular Jane Chiwoko, the lab technician Shameem Buleya, the laboratory staff at UNC Lilongwe Project, and finally the participat- ing patients.}

\section{Declarations}

Parts of the data were presented during the 12th International Workshop on HIV Treatment, Pathogenesis, and Prevention Research in Resource-limited Settings INTEREST 29 May -1 June 2018, Kigali, Rwanda, Abstract 181.

\section{Authors' contributions}

FN, AdF, EH, AN, CS, RK, HMS, HT, GF, SP. FN, SP, HT and GF conceived the study. $\mathrm{AdF}, \mathrm{AN}, \mathrm{HT}$ and SP were responsible for the local conduction and study logistics. EH, RK and CS advised on and conducted resistance analysis and interpretation. HT, AN, AdF, HMS and FN were involved in collection, cleaning and analysis of data; GF and SP provided study oversight. FN wrote the first draft, AdF provided statistical analysis, led the revision process and conducted final proof reading. All authors read and approved the final manuscript.

\section{Funding}

The study was funded through the grant M72 by Hector Stiftungen, Weinheim, Germany, recipient FN. The funder had no influence on the conduction or analysis of the study.

\section{Availability of data and materials}

Since the presented results are only part of the full study (see ClinicalTrials.gov NCT02381275), data can be made available upon specific request.

\section{Ethics approval and consent to participate}

The study protocol and procedures have been approved by the respective committees of Malawi and Germany. In particular the National Health Sciences Research Committee (NHSRC), Malawi Protocol Number \#1199 and the Ethic Committee of the Medical Faculty of the University Heidelberg, Protocol Number S-293/2014. All participants gave written consent.

\section{Consent for publication}

Not applicable.

\section{Competing interests}

The authors declare no conflict of interest.

\section{Author details}

${ }^{1}$ Heidelberg Institute for Global Health, University Hospital of Heidelberg, Im Neuenheimer Feld 130.3, 69120 Heidelberg, Germany. ${ }^{2}$ Institute of Virology, University of Cologne, Faculty of Medicine and University Hospital of Cologne, Cologne, Germany. ${ }^{3}$ Lighthouse Clinic, Lilongwe, Malawi. ${ }^{4}$ Institute of Virology and Immunobiology, University of Würzburg, Würzburg, Germany. ${ }^{5}$ Clinic for Gastroenterology and Hepatology, Faculty of Medicine and University Hospital of Cologne, Cologne, Germany. ${ }^{6}$ Department of Internal Medicine I, Faculty of Medicine and University Hospital of Cologne, Cologne, Germany. ${ }^{7}$ Department of Global Health, University of Washington, Seattle, WA 98104, USA. ${ }^{8}$ Department of Medicine, University of North Carolina School of Medicine, Chapel Hill, NC, USA. ${ }^{9}$ Department of Public Health, College of Medicine, School of Public Health and Family Medicine, University of Malawi, Zomba, Malawi.

Received: 3 March 2020 Accepted: 15 May 2020

Published online: 20 May 2020

\section{References}

1. Hemelaar J, Elangovan R, Yun J, et al. Global and regional molecular epidemiology of HIV-1, 1990-2015: a systematic review, global survey, and trend analysis. Lancet Infect Dis. 2019;19(2):143-55. https://doi. org/10.1016/S1473-3099(18)30647-9.

2. UNAIDS. UNAIDS data 2019. https://www.unaids.org/sites/default/files/ media_asset/2019-UNAIDS-data_en.pdf. Accessed 20 Jan 2020.

3. WHO. $\bar{W} H \mathrm{HO}$ drug resistance report 2017. https://www.who.int/hiv/pub/ drugresistance/hivdr-report-2019/en/. Accessed 20 Jan 2020.

4. Chimukangara B, Lessells RJ, Rhee S-Y, et al. Trends in pretreatment HIV-1 drug resistance in antiretroviral therapy-naive adults in South Africa, 2000-2016: a pooled sequence analysis. EClin Med. 2019;9:26-34. https:// doi.org/10.1016/j.eclinm.2019.03.006.

5. Inzaule SC, Hamers RL, Bertagnolio S, Siedner MJ, de Rinke Wit TF, Gupta RK. Pretreatment HIV drug resistance in low- and middle-income countries. Future Virol. 2019;14(6):427-40. https://doi.org/10.2217/ fvl-2018-0208.

6. Ministry of Health Malawi. Malawi_Integrated_HIV_Program_Report_2018_Q3_appendices.

7. Phiri S, Neuhann F, Glaser N, Gass T, Chaweza T, Tweya H. The path from a volunteer initiative to an established institution: evaluating 15 years of the development and contribution of the Lighthouse trust to the Malawian HIV response. BMC Health Serv Res. 2017. https://doi. org/10.1186/s12913-017-2466-y.

8. Lübke N, Di Cristanziano V, Sierra S, et al. Proviral DNA as a target for HIV-1 resistance analysis. Intervirology. 2015;58(3):184-9. https://doi. org/10.1159/000431093.

9. Sierra S, Dybowski JN, Pironti A, et al. Parameters influencing baseline HIV-1 genotypic tropism testing related to clinical outcome in patients on maraviroc. PLOS ONE. 2015;10(5):e0125502. https://doi.org/10.1371/ journal.pone.0125502.

10. Struck D, Lawyer G, Ternes A-M, Schmit J-C, Bercoff DP. COMET: adaptive context-based modeling for ultrafast HIV-1 subtype identification. Nucleic Acids Res. 2014;42(18):e144. https://doi.org/10.1093/nar/gku739.

11. Liu Tommy F, Shafer Robert W. Web resources for HIV type 1 genotypicresistance test interpretation. Clin Infect Dis. 2006;42:1608-18.

12. Hamers RL, Schuurman R, Sigaloff KCE, et al. Effect of pretreatment HIV-1 drug resistance on immunological, virological, and drug-resistance 
outcomes of first-line antiretroviral treatment in sub-Saharan Africa: a multicentre cohort study. Lancet Infect Dis. 2012;12(4):307-17. https:// doi.org/10.1016/\$1473-3099(11)70255-9.

13. Rutstein SE, Chen JS, Nelson JAE, Phiri S, Miller WC, Hosseinipour MC. High rates of transmitted NNRTI resistance among persons with acute HIV infection in Malawi: implications for first-line dolutegravir scale-up. AIDS Res Ther. 2019;16(1):5. https://doi.org/10.1186/s12981-019-0220-8.

14. ICAP. Malawi Population-based HIV Impact Assessment: MPHIA 20152016. [Summary Sheet]. https://phia.icap.columbia.edu/wp-content/ uploads/2018/10/MPHIA-SS_2018_FINAL.pdf. Accessed 20 Jan 2020.

15. Telele NF, Kalu AW, Gebre-Selassie S, et al. Pretreatment drug resistance in a large countrywide Ethiopian HIV-1C cohort: a comparison of Sanger and high-throughput sequencing. Sci Rep. 2018;8(1):7556. https://doi. org/10.1038/s41598-018-25888-6.
16. WHO. Gobal action plan on HIV drug resistance 2017-2021. https:// apps.who.int/iris/bitstream/handle/10665/255883/9789241512848-eng. pdf?sequence $=1$. Accessed 22 Jan 2020.

17. Dow DE, Bartlett JA. Dolutegravir, the second-generation of integrase Strand Transfer Inhibitors (INSTIs) for the treatment of HIV. Infect Dis Ther. 2014;3(2):83-102. https://doi.org/10.1007/s40121-014-0029-7.

\section{Publisher's Note}

Springer Nature remains neutral with regard to jurisdictional claims in published maps and institutional affiliations.
Ready to submit your research? Choose BMC and benefit from:

- fast, convenient online submission

- thorough peer review by experienced researchers in your field

- rapid publication on acceptance

- support for research data, including large and complex data types

- gold Open Access which fosters wider collaboration and increased citations

- maximum visibility for your research: over $100 \mathrm{M}$ website views per year

At BMC, research is always in progress.

Learn more biomedcentral.com/submissions 